\title{
Review of: "Rhizophora Mucronata Lam. (Mangrove) Bark Extract Prevents Ethanol-induced Liver Injury, Oxidative Stress and Apoptosis in Swiss Albino Mice"
}

Lionel Cyrille Bomgning Kamga

Potential competing interests: The author(s) declared that no potential competing interests exist.

\section{ABSTRACT AND INTRODUCTION}

The title "Rhizophora Mucronata Lam. (Mangrove) Bark Extract Prevents Ethanol-induced Liver Injury, Oxidative Stress and Apoptosis in Swiss Albino Mice" is not appropriate. Since liver injury, oxidative stress and apoptosis are separate, it means that BEARM possesses an intrinsic hepatoprotective effect. However, there is no assay in this work demonstrating that. Rather, this work shows that the hepatoprotective effect of BERM may be owed to antiOxidative and anti-apoptosis effects. So the title needs to be written appropriately.

In the abstract, look at the following sentence: "The ethanol intoxication caused large areas of liver "necrosis". The word "necrosis" here is not appropriate, I suggest you use "liver cell death". Necrosis and apoptosis are 2 mechanistically different types of cell death and it has been shown in this work that BERM possesses anti-apoptotic effect. So it is inappropriate to use necrosis here. You should therefore remove "necrosis" from the key words list. The same remark is observed in the results section.

It is not important in this work to mention the kidney in the abstract and the methodology and discussion since it has not been useful to carry out any assay and also, from the title, it is clear that the nephroprotection of BERM was not targeted. Please remove it from the work.

Free assertion with no support results: it is mentioned in the abstract and in this work that "BERM administration decreased ethanol-induced liver injury, as compared to the mice treated with ethanol alone", but there is no results demonstrating it. ALAT and ASAT assays are the best biochemical markers of liver toxicity or liver injury.

Alanine aminotransferase (ALT) and aspartate aminotransferase (AST) are the easiest and most important assays used to ratify both a substance-induced hepatotoxicity/liver damage and the hepatoprotective effect of interest compounds or plants. Without those two assays, how can you be sure of alcohol hepatotoxicity and BERM preventive hepatoprotection effect? Those two tests are crucial to validate this work. 
In the whole document, remove "extract" in front of the abbreviation "BERM" since "E" already means "extract"

In the introduction, the following sentence "This study primarily focused on the evaluation of liver injury biomarkers, including the measurements of nonenzymatic antioxidant components, MDA and GSH, using oxidative stress-related biochemical assays" is not understandable. From this sentence it is clear that the title needs to be rephrased.

Why have you evaluated TNF- $\alpha$ gene expression in this work? TNF- $\alpha$ is usually used to appraise the level of inflammation in an organism. Was it the case here?

\section{MATERIALS AND METHODS}

You can't say that animals were euthanized since they were not suffering. They were rather sacrificed. So replace euthanized by sacrificed.

\section{RESULTS}

\subsection{BERM Prevents Ethanol Intoxication-Induced Liver Injury}

Remove the kidneys and the results concerning kidneys in all the work, it is not relevant. If you really want to include it in the work completely change the title of the article by adding "nephroprotection".

The images on the figure 2 are not well appreciated. It is difficult to observe both damages caused by alcohol administration and the protective effect of BERM on liver architecture. Rather of a protective effect of BERM, I am observing a worse effect since congestion, ballooning can be clearly observed. There is also no regenerative cells as stated.

Also the stated "necrosis" here is not sufficient to conclude on BERM hepatoprotection. There are other factors used in hisptopathological analysis like the congestion of centrilobular vein, the ballonning, neutrophil infiltration, ...

You can't be concluded on fibrosis In this work since it was not evaluated. There are several ways to diagnose fibrosis in liver cells like "Collagen Deposition", "Hepatic Stellate Cell Activation (evaluating TGF$\beta 1$ contents of serum", "Immunohistochemical analysis of $\alpha$-SMA". Also, to detect the distribution of fibrotic tissue, Masson staining and Sirius red staining is generally applied on liver tissue.

\subsection{BERM Decreases Ethanol-Induced MDA Levels and Enhances Ethanol-decreased GSH Levels in Liver}

Tissues

Write "lipid peroxidation" and not "liperoxidation".

In the entire results part, there is a great emphasis on the effect of silymarin as if it was a tested compound of interest whereas it is only used here as a positive control. I suggest you put more emphasis on the effect of BERM and just use the effect of silymarin to back up.

The normal control group here should be used in comparison to show the bad effect of alcohol administration. 
TNF- $\alpha$ Gene Expression Level measurements is generally used to demonstrate an anti-inflammatory effect of the substance of interest. What about this work?

The following sentence: "The apoptotic index was significantly decreased in ethanol-induced liver injury in the mice group pre-treated with BERM", is not written appropriately. You should say: The apoptotic index was significantly decreased by BERM pre-treatment. 